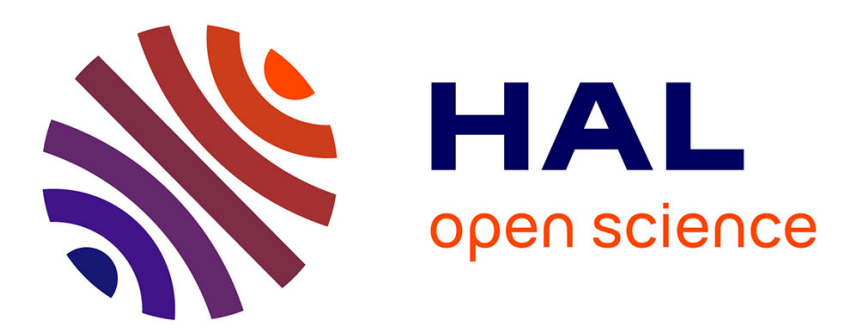

\title{
A Methodology for Classifying Operating Regimes in Underbalanced-Drilling Operations
}

Ulf Jakob F. Aarsnes, Florent Di Meglio, Robert Graham, Ole Morten Aamo

\section{To cite this version:}

Ulf Jakob F. Aarsnes, Florent Di Meglio, Robert Graham, Ole Morten Aamo. A Methodology for Classifying Operating Regimes in Underbalanced-Drilling Operations. Society of Petroleum Engineers Journal, 2016, 21 (02), pp.423-433. 10.2118/178920-PA . hal-01544668

HAL Id: hal-01544668

https://hal-mines-paristech.archives-ouvertes.fr/hal-01544668

Submitted on 3 Sep 2020

HAL is a multi-disciplinary open access archive for the deposit and dissemination of scientific research documents, whether they are published or not. The documents may come from teaching and research institutions in France or abroad, or from public or private research centers.
L'archive ouverte pluridisciplinaire HAL, est destinée au dépôt et à la diffusion de documents scientifiques de niveau recherche, publiés ou non, émanant des établissements d'enseignement et de recherche français ou étrangers, des laboratoires publics ou privés. 


\title{
A Methodology for Classifying Operating Regimes in Underbalanced-Drilling Operations
}

\author{
Ulf Jakob F. Aarsnes, Norwegian University of Science and Technology, \\ Florent Di Meglio, MINES ParisTech, Robert Graham, Well Advanced Solutions, and Ole \\ Morten Aamo, Norwegian University of Science and Technology
}

\begin{abstract}
Summary
This paper proposes an extension to an existing operating-envelope technique used for underbalanced drilling (UBD) to enhance control of bottomhole pressure and inflow parameters. With the use of an implementation of the drift-flux model (DFM) with boundary conditions typically encountered in underbalanced operations (UBO), a steady-state analysis of the system is performed. Through this analysis, four distinct operating regimes are identified, and the behavior in each of them is investigated through steady-state calculations and transient simulations. In particular, the analysis reveals that a section of the operating envelope previously believed to be unstable/transient is, in fact, stable/steady when a fixed choke opening is used as an independent variable in place of a fixed wellhead pressure (WHP). This results in the steady-state operating envelope being extended, and gives an increased understanding of the well behavior encountered in UBO toward enabling the introduction of automated control. Finally, we investigate the mechanism behind severe slugging in UBO and argue that the cause is different from that of the slugging encountered in production and multiphase transport.
\end{abstract}

\section{Introduction}

Note that, in recent years, an increasing degree of automation in managed-pressure drilling occurred (Thorogood et al. 2010). The pay-off was increased safety, as well as enabling the drilling of previously undrillable wells, because of tighter control of downhole pressure (Godhavn 2010, 2011). It is natural to believe that we will see a similar development toward increased automation in UBO.

Unfortunately, in the context of automated pressure and flow control, the dynamics of the two-phase flow encountered in UBO is significantly more complicated than the single-phase flow of conventional drilling: In single-phase flow, any operating point is inherently stable, transients are short and predictable, and, barring certain well-control incidents, operating conditions are reasonably homogeneous. By contrast, in two-phase UBO, the distributed gas/liquid flow and the reservoir/well interaction result in classical nonlinear behavior [see Khalil (2002) for description of nonlinear behavior of dynamics systems] such as multiple steady states, limit cycles, and bifurcations, as described by Aarsnes et al. (2014a, b) and Mykytiw et al. (2003, 2004). Hence, to enable safe and robust algorithms for automated control to be developed for UBO, increased understanding of these phenomena, their potential occurrence in UBO, and the behavior of the coupled wellreservoir dynamics are required.

Contribution. The main contribution of this paper is the classification of operating points in UBO. The associated analysis performed to attain this classification also yields understanding of the behavior of the system.
More precisely, a comprehensive steady-state analysis of a DFM coupled with a reservoir is performed. The dynamics of this model replicates dynamics that are encountered in UBO in gasdominant reservoirs. Investigating the steady states of a model also reveals much of the transient behavior.

To this end, we present an extension to the operating-envelope analysis technique presented by Graham and Culen (2004) and used by Mykytiw et al. (2004), which have properties that are particularly beneficial from a pressure-control point of view compared with the conventional UBO operating-envelope analysis technique, used, for example, in Saponja (1998) and Guo and Ghalambor (2002).

This new technique, combined with analysis tools from dynamic-systems theory, is used to investigate when the flow in the well is stable/steady and when it might become unstable. In particular, it is shown that whether a well is hydrostatic or friction-dominated [in the sense used by Saponja (1998)] is not the determining factor for flow stability in UBO. Instead, a new classification of the UBO envelope is proposed in which the part with stable flow can be identified as either an "intuitive regime" with short and well-behaved transient dynamics or a "nonintuitive regime" with an inverted response in the WHP. In addition, an "unstable regime" with no stable steady states, an operating region with potential slugging, and finally, conventional overbalanced drilling round out the classification to five distinct regimes.

Outline. In the next section, some concepts from dynamic-systems theory are introduced that will be helpful in the later discussion on the dynamics. Next, the DFM at steady state is described, with the full model given in Appendix A. With a short transient simulation case, an alternative operating-envelope analysis technique is hypothesized and then proposed. This is then used to identify and classify five distinct operating regimes in UBO, when including severe slugging as a distinct regime (because the potential occurrence of severe slugging is not captured directly by this technique, it is given its own treatment). This is the main result of the paper. We end the paper with a Conclusion section and some thoughts on the potential of automatic control in light of the preceding analysis.

\section{Stable and Unstable Equilibria of Dynamical Systems}

This section introduces some well-known concepts and results from the field of dynamic-systems theory that will be useful in the following discussion. For a more comprehensive treatment, the interested reader is referred to Khalil (2002).

Consider the autonomous system

$$
\dot{x}=f(x), \ldots \ldots \ldots \ldots \ldots \ldots \ldots \ldots \ldots \ldots \ldots \ldots \ldots
$$

where $x$ is a vector of system states and $\dot{x}$ denotes the time derivative of these states such that the dynamics of the system are described by the function $f(x)$. A state $\bar{x}$ is an equilibrium of Eq. 1 if it satisfies $f(\bar{x})=0$. The following definition will be of importance. 


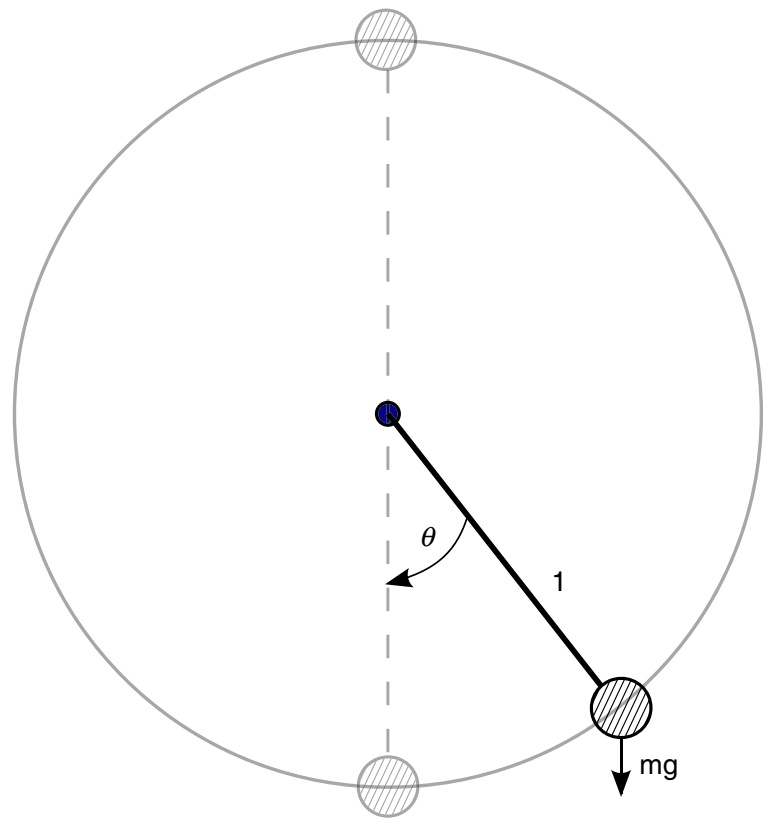

Fig. 1-Pendulum.

Definition 1. The equilibrium point $\bar{x}$ of Eq. 1 is

- stable if, for each $\varepsilon>0$, there is $\delta=\delta(\varepsilon)>0$ such that

$\|x(0)-\bar{x}\|<\delta \Rightarrow\|x(t)-\bar{x}\|<\varepsilon, \quad \forall t \geq 0 \quad \ldots \ldots$

- unstable if it is not stable

- asymptotically stable if it is stable and $\delta$ can be chosen such that

$$
\|x(0)-\bar{x}\|<\delta \Rightarrow \lim _{t \rightarrow \infty} x(t)=\bar{x} .
$$

The implication of this definition is that a system will diverge from an unstable equilibrium, remain close to a stable equilibrium, and converge to an asymptotically stable equilibrium. The following example is illustrative.

Example. Consider the pendulum system shown in Fig. 1. The pendulum equation can be derived using Newton's second law of motion and written as

$$
\begin{aligned}
& \dot{\theta}_{1}=\omega \ldots \ldots \ldots \ldots \ldots \ldots \ldots \ldots \ldots \ldots \ldots \ldots \ldots \ldots \ldots \ldots \ldots \ldots \\
& \dot{\omega}_{2}=-\frac{g}{l} \sin (\theta)-\frac{k}{m} \omega, \ldots \ldots \ldots \ldots \ldots
\end{aligned}
$$

where $\theta, \omega$ denotes the angular position and velocity and $k$ is the frictional coefficient. This system has two equilibrium points $\theta=0, \omega=0$ and $\theta=\pi, \omega=0$. Starting in an equilibrium point, the system will remain stationary, i.e. at steady state. However, for the second equilibrium point at $\theta=\pi$, all trajectories starting arbitrarily close will eventually diverge and leave the ball $\|x-\bar{x}\| \leq \varepsilon$. This point clearly does not satisfy the $\varepsilon-\delta$ requirement for stability and is hence an unstable equilibrium point. Unstable points are not uninteresting, however, since they can be rendered stable by feedback control and yield insight into the behavior of the system. The equilibrium at $\theta=0$ is asymptotically stable as nearby trajectories will not only remain close, thus satisfying the $\varepsilon-\delta$ requirement, but will converge to the equilibrium point as time tends to infinity because of frictional damping.

Analysis Through Linearization. A well-known technique in analyzing nonlinear systems is to exploit the fact that, close to an equilibrium, a systems behavior matches closely to that of its linear approximation. In fact, the stability of an equilibrium point can, in most instances, be determined by checking the stability of the linearized system, as per Lyapunov's indirect method (Khalil
2002). This is usually performed by using a classic result that states that a linear system is unstable if one or more of its poles (i.e., zeros of the system's characteristic equation) is in the righthalf plane (i.e., has a positive real part) (Aström and Murray 2010). The qualitative behavior of the linear system can be further characterized according to the location of the unstable poles in the right-half plane:

1. Pole on the real axis in the right-half plane: simple exponential divergence.

2. Complex conjugate pair of poles in the right-half plane: oscillations with exponentially increasing amplitude.

In the present case, the analysis is complicated by the fact that the DFM is a distributed parameter system (i.e., described by a partial-differential equation), which means it has an infinite number of modes. Thus, an equilibrium point can have multiple poles in the right-half plane, and firm predictions become difficult to make. We will, however, in this paper, try to draw conclusions on the behavior on the basis of a combination of mathematical analysis and simulations. In this regard, we will distinguish between two instabilities on the basis of qualitative behavior observed in simulations:

1. Static instability: believed to be caused by the equilibrium dominated by a pole on the real axis in the right-half plane. It exhibits a simple exponential divergence from the equilibrium. It can be identified as an unstable equilibrium by the stability heuristic presented later in the paper.

2. Dynamic instability: believed to occur when the equilibrium is unstable because of a complex conjugate pair of poles in the right-half plane whereas, at the same time, there is no pole on the real axis in the right-half plane. It is characterized by an oscillation around the equilibrium point with an exponentially increasing magnitude. The absence of the pole on the real axis means that this instability is not identified by the stability heuristic. It can, however, be identified through simulations and the Nyquist stability criterion.

By identifying all equilibriums of a system and determining their properties through linear analysis, one can make predictions about the full nonlinear dynamics as well. However, these predictions are only valid close to the equilibrium, and the behavior may radically change when the system significantly diverges away from it. Thus, we combined the predictions from the analysis of the linearized equilibrium points with transient simulations to substantiate a more comprehensive understanding of the full system dynamics.

\section{The DFM at Steady State}

To model the pressure and flow dynamics in the well, we will use the DFM, which is a frequently used model of multiphase flow in drilling. The DFM requires one distributed state for each phase to model the mass balance while the momentum of the mixture is lumped into one equation. Seminal references on two-phase flow and the DFM are Wallis (1969) and Ishii (1977).

In the context of drilling, seminal work was performed by Lage et al. (2000), Lage and Time (2002), and Fjelde et al. (2003) to validate both the steady-state and transient behavior of the DFM with full-scale experimental data. These studies show that the basic DFM yields good qualitative predictions, and that a high quantitative accuracy can be achieved as well when using flowpattern identification with separate closure relations for each flow pattern. In this work, we avoid this final complication by using a DFM with simple friction and slip correlations, independent of flow patterns. This simplifies the analysis of the model while retaining the qualitative behavior.

A description of the full DFM is given in Appendix A. What follows is the boundary conditions and steady-state version of the DFM equations, which are used to calculate the operating envelope. Description of dependent variables is given in Table $\mathbf{1}$ and parameters in Table 2.

Boundary Conditions. Let $x \in[0, L]$ denote the space variable. For clarity purposes, we denote the pressure at the boundaries 


\begin{tabular}{cc|cc}
\hline$n=\alpha_{G} \rho_{G}$ & Gas mass variable & $m=\alpha_{L} \rho_{L}$ & Liquid Mass Variable \\
$W_{G}=A n v_{G}$ & Gas mass-rate & $W_{L}=A m v_{L}$ & Liquid mass-rate \\
$\alpha_{G}$ & Void fraction & $\alpha_{L}$ & Liquid holdup \\
$\rho_{G}$ & Gas density & $\rho_{L}$ & Liquid density \\
$v_{G}$ & Gas velocity & $v_{L}$ & Liquid velocity \\
$v_{M}$ & Mixture velocity & $P$ & Distributed pressure \\
\hline
\end{tabular}

Table 1-List of dependent variables.

\begin{tabular}{lccc} 
Description & Symbol & Value & Unit \\
\hline Area of flow & $A$ & $6.8 \times 10^{-3}$ & $\mathrm{~m}^{2}$ \\
Gas-production index & $k_{G}$ & $5 \times 10^{-7}$ & $\mathrm{~kg} / \mathrm{s} / \mathrm{Pa}$ \\
Liquid-production index & $k_{L}$ & 0 & $\mathrm{~kg} / \mathrm{s} / \mathrm{Pa}$ \\
Slip parameter & $K$ & 1.5 & - \\
Slip parameter & $S$ & 1.0 & $\mathrm{~m} / \mathrm{s}$ \\
Inclination & $\phi(x)$ & $\pi / 2$ & - \\
Measured depth & $L$ & 2530 & $\mathrm{~m}$ \\
Reservoir pressure & $P_{\text {res }}$ & 279 & $\mathrm{bar}$ \\
Reference liquid density & $\rho_{0, L}$ & 1000 & $\mathrm{~kg} / \mathrm{m}^{3}$ \\
\hline
\end{tabular}

Table 2-List of parameters.

as $P(x=0)=B H C P$, where $B H C P$ is the bottomhole circulating pressure, and $P(x=L)=W H P$ (Fig. 2). Boundary conditions on the downhole boundary are given by the mass rates of gas and liquid injected from the drilling rig and flowing in from the reservoir. Denoting the cross-sectional flow area by $A$, the boundary fluxes are given as:

$$
\begin{aligned}
& \left.A m v_{L}\right|_{x=0}=k_{L} \max \left(P_{\text {res }}-B H C P, 0\right)+W_{L, \text { inj }}(t), \ldots \\
& \left.A n v_{G}\right|_{x=0}=k_{G} \max \left(P_{\text {res }}-B H C P, 0\right)+W_{G, \text { inj }}(t) . \ldots
\end{aligned}
$$

Here $P_{\text {res }}$ denotes the reservoir pore pressure and $k_{G}, k_{L}$ are the production index of the gas and liquid, respectively. The injection

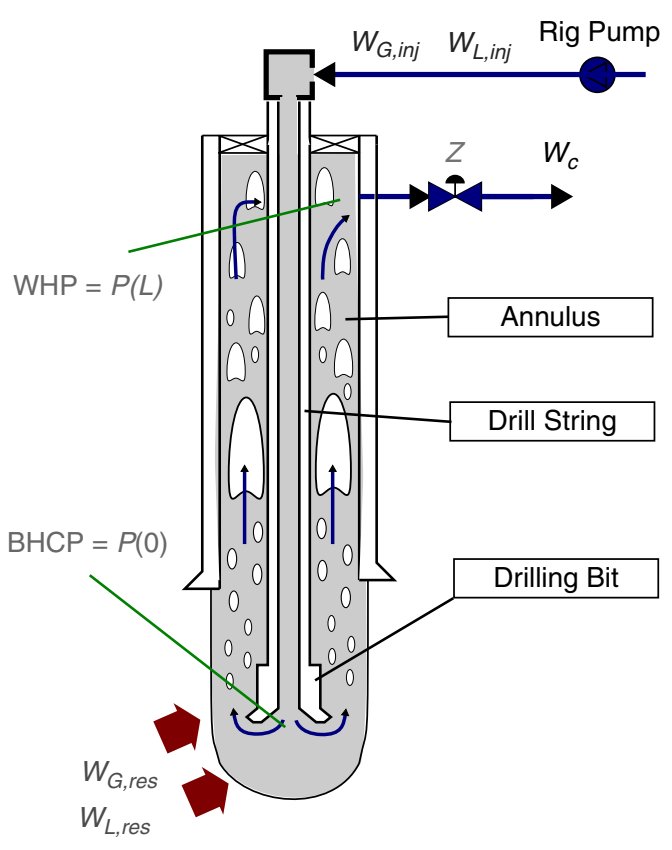

Fig. 2-UBD schematic. mass-rates of gas and liquid, $W_{G, \text { inj }}, W_{L, \text { inj }}$, are specified by the driller and can, within some constraints, be considered as manipulated variables. The inflow from the reservoir is dependent on the pressure on the left boundary, usually given by a Vogel-type inflow performance relationship (IPR) (Wiggins et al. 1996), but within the operational range of a typical UBD operation; a linear approximation should suffice.

We consider two different topside boundary conditions, corresponding to two potential operating scenarios:

1. The WHP is set to be constant:

$$
\text { WHP }=\text { const } .
$$

2. WHP is dependent on the topside liquid and gas mass-rates through a valve equation:

$$
\frac{m v_{L}}{\sqrt{\rho_{L}}}+\left.\frac{n v_{G}}{Y \sqrt{\rho_{G}}}\right|_{x=L}=\frac{C_{v}[Z(t)]}{A} \sqrt{W H P-P_{s}},
$$

where $C_{v}$ is the choke opening given by the manipulated variable $Z . Y \in[0,1]$ is a gas-expansion factor for the gas flow, and $P_{s}$ is the separator pressure (i.e., the pressure downstream from the choke).

Steady-State Equations. The model is said to be at an equilibrium, or at steady state, when both the boundary conditions Eqs. 6 and 7 and Eq. 9 or Eq. 8 and the distributed Eqs. 26 through 28 with the $\frac{\partial}{\partial t}$ terms to zero are satisfied. That is, at steady state, we have

$$
\begin{aligned}
& \frac{\partial m v_{L}}{\partial x}=0 \\
& \frac{\partial n v_{G}}{\partial x}=0 \\
& \frac{\partial P+m v_{L}^{2}+n v_{G}^{2}}{\partial x}=-(m+n) g \sin \phi(x)-\frac{2 f(m+n) v_{m}\left|v_{m}\right|}{D} .
\end{aligned}
$$

From Eqs. 10 and 11, we have that the mass flux is constant with regards to the variable $x$. Combining this with the boundary conditions and integrating Eq. 12, we find that the system at steady state must satisfy

$$
\begin{aligned}
W H P= & B H C P+\int_{0}^{L}-\underbrace{\frac{\partial m v_{L}^{2}+n v_{G}^{2}}{\partial s}}_{\text {Acceleration }}-\underbrace{(m+n) g \sin \phi(s)}_{\text {Gravity }} \\
& -\underbrace{\frac{2 f(m+n) v_{m}\left|v_{m}\right|}{D} \mathrm{~d} s, \ldots \ldots \ldots \ldots}_{\text {Friction }} . \ldots \ldots \\
A m v_{L}= & k_{L} \max \left(P_{\text {res }}-B H C P, 0\right)+W_{L, \text { inj }}(t), \ldots \ldots \\
A n v_{G}= & k_{G} \max \left(P_{\text {res }}-B H C P, 0\right)+W_{G, \text { inj }}(t), \ldots \ldots .
\end{aligned}
$$

and the topside boundary condition Eq. 8 or 9 . 


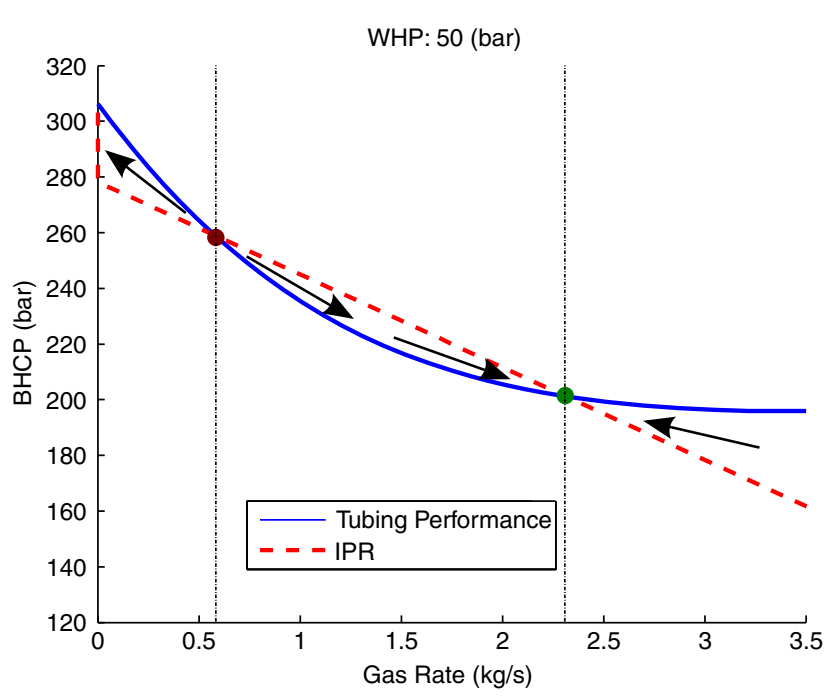

Fig. 3-Stable and unstable equilibrium in a UB operation.

\section{UBD Operating Envelope}

In UBD well engineering, using a steady-state multiphase flow simulator is a popular approach to develop an operating envelope, or operating window. This allows the engineer to gauge the WHP and injection rates required to achieve the desired BHCP as well as satisfying holecleaning requirements. Publications referring to the use of such a technique are also numerous; see, for example, Saponja (1998), Guo and Ghalambor (2002), Udegbunam et al. (2013), Nguyen et al. (2009), Suryanarayana et al. (2006), Guo (2002), and Pickles et al. (2004).

In the following, we consider an example case to illustrate the failure of the conventional operating envelope to predict stability of certain equilibria caused by the assumption of constant WHP. An alternative approach that amends this problem is then proposed. For the case considered, no gas injected and no liquid produced (i.e., a constant liquid rate and pressure-dependent gas rate) is assumed. See Table 2 for full list of parameter values used.

Conventional Operating-Envelope Analysis. The conventional way of developing the operating envelope is to assume a fixed WHP, thus satisfying Eq. 8, and then specify a gas mass-rate $n v_{G}$ and solve Eqs. 13 and 14 to find the corresponding BHCP. This is then repeated over a range of gas mass-rates, thus creating the tubing performance curve (see Fig. 3). This curve gives the potential steady-state operating points of the well. To find the actual equilibria of the system, we need to overlay the solution of Eq. 15, which is the IPR curve. The intersection points of these two curves correspond to an equilibrium, because it indicates that the

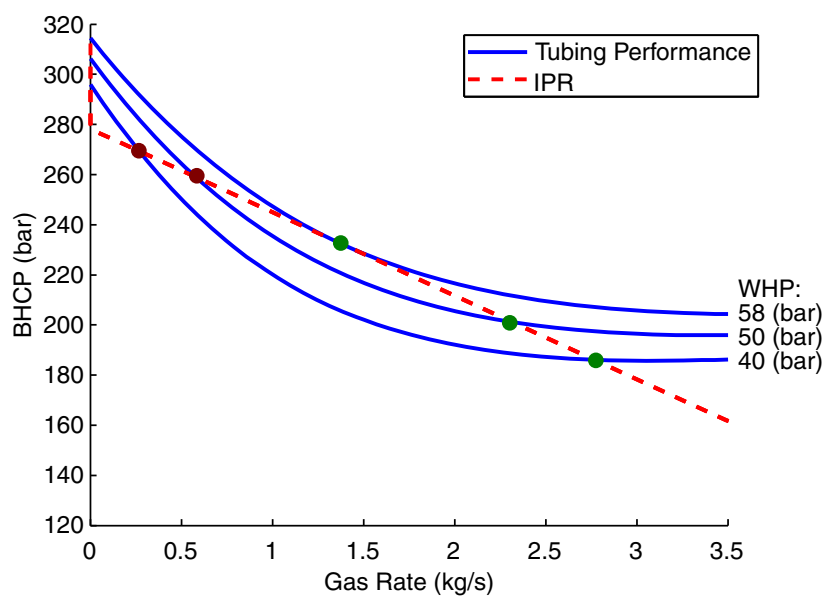

Fig. 5-Highest achievable BHCP with a constant WHP.

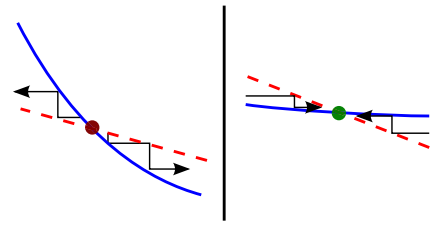

Fig. 4-Dynamics of an unstable and a stable equilibrium.

full set of Eqs. 8, 13 through 15 are satisfied. These equilibria can be either stable or unstable; see Definition 1.

In Fig. 3, there are two intersection points, but only one of these is stable and a suitable operating point. By inspecting the curves, we see that, for gas rates in which the IPR curve is above the tubing performance curve, the well is not in a steady state but tends to a higher gas rate and vice versa (see Fig. 4). The intersection point denoted by the red dot, then, is unstable: A slight perturbation from this point to an increased BHCP would cause a reduced gas inflow, further increasing the $\mathrm{BHCP}$. Vice versa, for a slight decrease in BHCP, the increased gas-influx will displace the liquid in the well, further decreasing BHCP and causing yet more gas-influx. Hence, this equilibrium has a static instability from which nearby trajectories will diverge.

For the steady state denoted by the green dot, the intersection between the two curves is in the opposite direction, making this an attractive (i.e., stable) equilibrium.

In the field, changing BHCP is typically achieved by controlling the WHP. One can see the effect of changing the WHP for the current scenario in Fig. 5, where the well will tend to the intersection denoted by the green dot for the tubing performance curve corresponding to the current WHP. Hence, according to this analysis, the highest underbalanced BHCP at a stable steady state that can be achieved for this well by changing the WHP is 235 bar, achieved with a WHP of 58 bar. Enforcing a WHP higher than this will lead to well becoming overbalanced.

Alternative Technique. In this section, we present an alternative approach to finding equilibria in UBO. The basis is similar to the techniques used by Mykytiw et al. (2004) and Graham and Culen (2004), but where these papers concluded that (what we call) the nonintuitive regime is unstable, we show that this is true only when using a constant WHP (i.e., Eq. 8) as a boundary condition. When we control by setting a fixed choke opening instead (i.e., using Eq. 9), the stable regime is expanded, as is shown in the following.

Transient Simulation. To give an impression of the thinking behind this approach, consider a transient simulation of the same well studied in the previous section but with the topside boundary condition Eq. 9 (see Fig. 6). Again, the injected liquid rate is kept constant, the gas rate is dependent on the BHCP according to Eq. 15 , and the only manipulated variable is the choke opening. Note that the effect of changing the choke opening is to change the WHP required to have a given mass-rate flowing out of the well, as given by Eq. 9 .

Initially, the choke opening is set to a (Table 3) and is changed (slightly closed) to $\mathbf{b}$, $\mathbf{c}$ and $\mathbf{d}$ after 3,6, and 10 hours. When the choke opening is changed, there is a transient period before the pressure settles to the new steady state. We note the qualitatively different responses for each of the changes in choke opening. For the change from a to $\mathbf{b}$, both the WHP and BHCP increases because the choke opening is decreasing as expected. For the decrease in choke opening from $\mathbf{b}$ to $\mathbf{c}$, however, after an initial increase in WHP, a counterintuitive response follows, in which the WHP decreases and settles at a lower value than the previous equilibrium. Finally, when the choke opening is set to d after 10 hours, the system drifts to overbalance.

Steady-State Analysis. It is clear that this transient behavior cannot be fully understood by the conventional operating-envelope analysis, and the reason for this is the failure of that method to take the effect of the backpressure choke, Eq. 9, into account. Specifically, the conventional operating-envelope analysis 

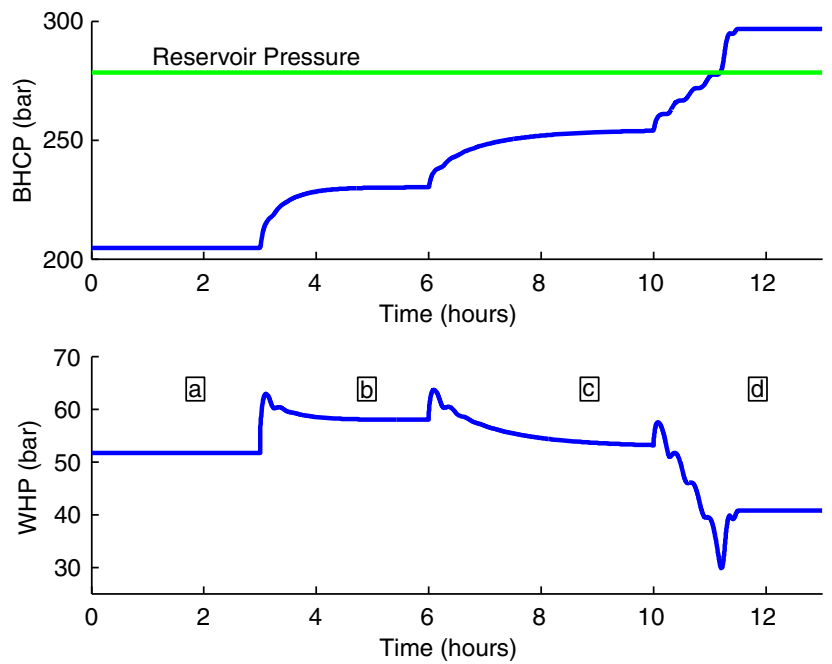

Fig. 6-Transient simulation with decreasing choke opening.

considers the WHP as the independent (i.e., exogenous) variable when in practice; the choke opening is the independent variable, which allows for including Eq. 9.

Instead, to understand this behavior, we return to the steadystate equations of the DFM, but, following the suggestion of Graham and Culen (2004), we combine the tubing performance curve and the IPR to plot the curve shown in Fig. 7. The blue WHP curve in this figure is calculated by fixing the BHCP, figuring out the inflow rates with Eqs. 14 and 15, and then finding the corresponding WHP by integrating Eq. 13. Equilibria can then be found by overlaying either Eq. 8 (which corresponds to Fig. 7) or Eq. 9 (corresponding to Fig. 8) according to the boundary condition that is enforced.

By comparing Fig. 7 with Fig. 5, it is seen that the same equilibria points are identified by both approaches. However, to understand the behavior from the transient simulation, the boundary condition with the backpressure choke, that is, Eq. 9, must be enforced. Overlaying Eq. 9 with the values in Table 3 yields Fig. 8 . Again, for certain choke openings, such as c, there are multiple points of intersection between the curves and correspondingly multiple equilibria. These can be checked for static instabilities by a "stability heuristic" similar to the one used for the conventional technique: When the red line is below the blue line, there is more mass flowing out of the well than into the well, and BHCP will decrease and vice versa. Hence, we can identify the qualitative behavior of the system and heuristically determine the stability or instability of the equilibria; see Fig. 9. In this figure the behavior of the well is also indicated by the arrows: If the well is started with a BHCP lower than that which corresponds to the unstable equilibrium, the red curve is below the blue, and the system moves toward the underbalanced stable equilibrium indicated by the green dot. Note that this stability heuristic is named as such because it is not rigorous; in fact, it is only able to detect static instabilities.

Now, consider the transient simulation (Fig. 6) in the context of Fig. 8: We see that, moving from choke opening $\mathbf{a}$ to $\mathbf{b}$, we have an intuitive response as the system moves to the apex of the steady-state WHP curve. Moving to choke opening c, however, results in a decreased steady-state WHP, as indicated by Fig. 8, which gives the nonintuitive transient response observed in Fig. 6. Finally, at $\mathbf{d}$, there are no intersections between the two curves at underbalanced BHCPs, and since the red curve is above the blue one, the well moves to the equilibrium in overbalanced conditions.

\section{Classification of Operating Regimes}

With the techniques presented in the previous section, four distinct regimes for the UBD well in question can be identified; see Fig. 10. In addition, there is a fifth regime caused by a dynamic instability that is characterized by a severe slugging limit cycle. This is investigated in a later section.

(1) Intuitive Regime. This regime corresponds to BHCPs below (i.e., left in Fig. 10) the apex of the WHP curve. The well in this regime is stable and well-behaved without exhibiting inverse responses.

(2) Nonintuitive Regime. In this regime the well exhibits inverse response in the WHP compared with BHCP. To explain this phenomenon, consider the following approximation of Eq. 13, obtained by neglecting the acceleration term:

$B H C P=F+G+W H P$,

where $F$ denotes the integrated frictional pressure drop and $G$ the hydrostatic pressure. Let $\Delta$ denote a steady-state change in value. We have

$$
\begin{aligned}
& \triangle W H P=\triangle B H C P-\Delta F-\Delta G, \ldots \\
& \Rightarrow \frac{\Delta W H P}{\triangle B H C P}=1-\frac{\Delta F}{\Delta B H C P}-\frac{\Delta G}{\Delta B H C P} .
\end{aligned}
$$

Inserting Eq. 15 in Eq. 18 results in

$$
\frac{\Delta W H P}{\Delta W_{G}}=-\frac{1}{k_{G}}-\frac{\Delta F}{\Delta W_{G}}-\frac{\Delta G}{\Delta W_{G}} .
$$

Hence, we get the nonintuitive response when (unfortunately, this criterion requires a steady-state model of the well to find $\frac{\Delta F}{\Delta W_{G}} ; \frac{\Delta G}{\Delta W_{G}}$, and cannot be computed explicitly from well parameters alone)

$\frac{1}{k_{G}}+\frac{\Delta F}{\Delta W_{G}}+\frac{\Delta G}{\Delta W_{G}}<0$,

and the apex of the curve in Fig. 10 corresponds to the point when the left side of Eq. 20 equals zero. Note that this condition is different from the one typically used to identify a hydrostatically dominated well, which is

$$
\begin{aligned}
& \frac{\Delta(B H C P-W H P)}{\Delta W_{G}}<0 \\
& \Rightarrow \frac{\Delta F}{\Delta W_{G}}+\frac{\Delta G}{\Delta W_{G}}<0 .
\end{aligned}
$$

We emphasize that the determining factor for the behavior of the well is the condition presented by Eq. 20 and not Eq. 22 .

In this nonintuitive regime, the pressure settles at a lower value, but since the system's initial response is an increase (see Fig. 6), we get an inverse response in the WHP. This phenomenon is referred to as the system being nonminimum phase in systems theory. Because of this nonminimum-phase response, the BHCP should not be controlled on WHP in this regime. This is why steady states in this regime appear unstable when the conventional technique shown in Fig. 5 is used. In this regime, BHCP should be controlled on choke opening.

(3) Unstable Regime. For larger values of BHCP, there are no stable equilibria. Closing the choke further, for

\begin{tabular}{ccccc}
\hline Time: & $1-3$ (hours) & $3-6$ (hours) & $6-10$ (hours) & $10-12$ (hours) \\
Choke Opening: & $\mathbf{a}=12 \%$ & $\mathbf{b}=10 \%$ & $\mathbf{c}=8.5 \%$ & $\mathbf{d}=7 \%$ \\
\hline
\end{tabular}

Table 3-Choke stepping program. 


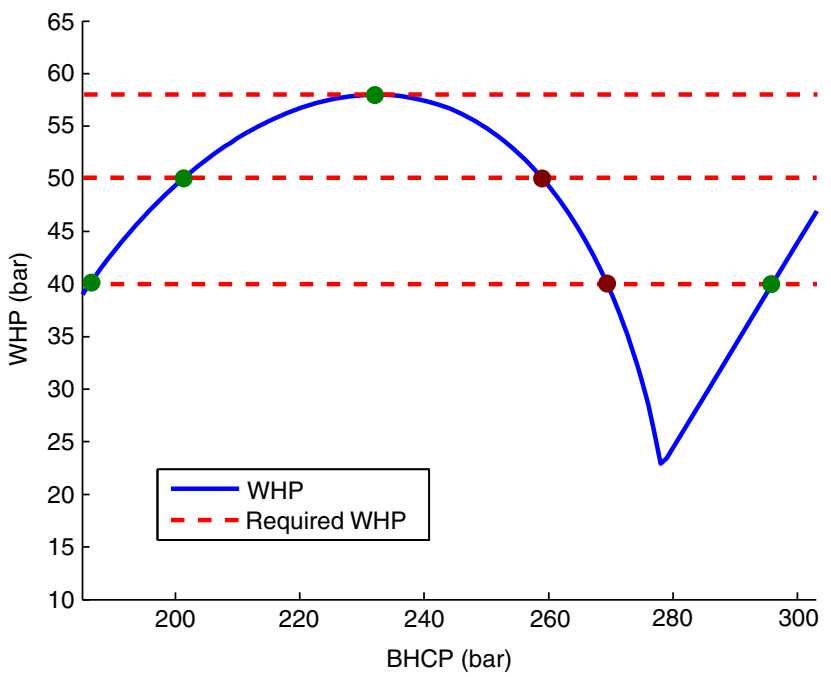

Fig. 7-Operating-envelope technique, as suggested by Graham and Culen (2004).

example, from $\mathbf{c}$ to $\mathbf{d}$, will cause the well to enter a limit cycle of severe slugging or drift toward the steady state in overbalanced conditions.

We note, however, that there are nominally unstable equilibria in this regime that can be made stable through automatic feedback control of the choke with BHCP measurements, that is, by creating the appropriate dynamic mapping $Z(t)=C(B H C P)$. Thus, the UBD operating envelope can be extended, which would enable performing UBO on wells with tight margins between fracture and collapse pressure or with stringent limits on the amount of gas that can be flared.

(4) Overbalanced Regime. In this regime, for the given well, the system contains only liquid, which makes the difference $B H C P-W H P$ constant. Hence, all steady states are stable with short predictable transients.

Identifying Operating Regime. As was shown, the operating envelope can be extended with the choke opening as the independent variable in place of WHP. While keeping the well under control in this manner, the well's operating regime can be identified by reducing the choke opening and observing the corresponding change in WHP (see Fig. 6). In the intuitive region far from the apex of the WHP curve (see Fig. 10), the well quickly goes to steady state after the change, without any overshoot and with an increase in steady-state WHP. Closer to the apex of the WHP

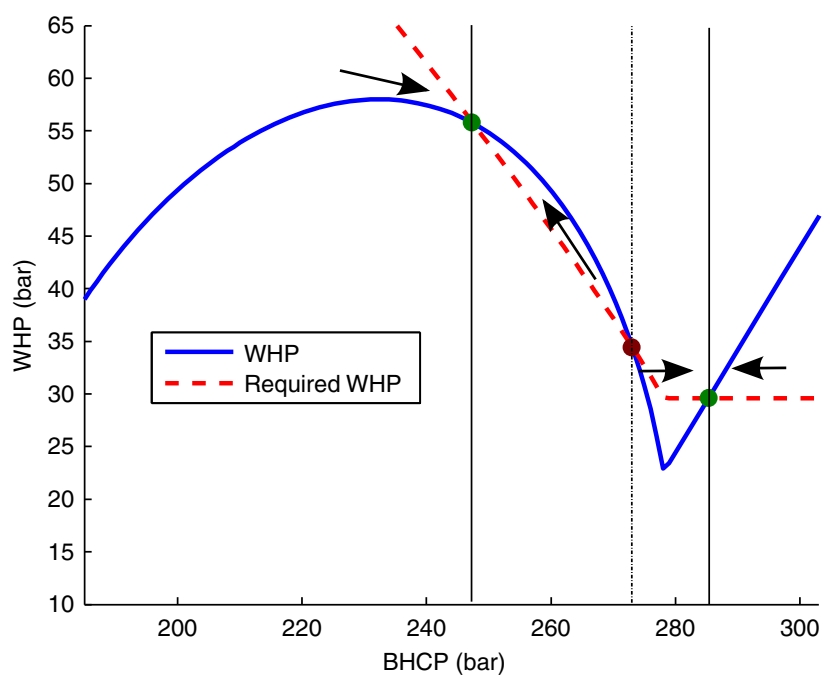

Fig. 9-Characterizing equilibria and transient response.

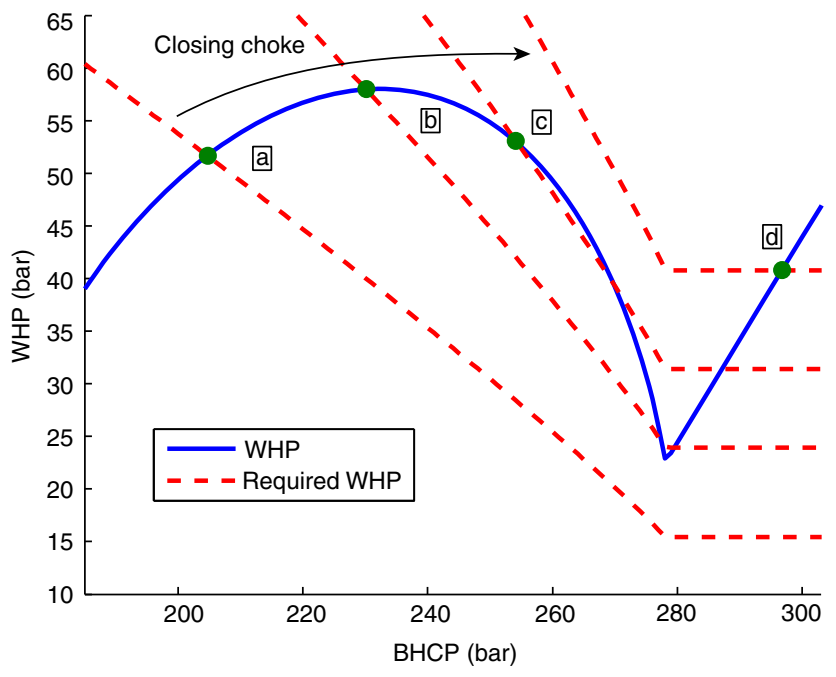

Fig. 8-Effect of using a valve equation at the boundary.

curve (and thus closer to the limit to the nonintuitive region), the well will need more time to reach steady state, and the WHP response will have an overshoot. In the nonintuitive regime, the WHP will have an inverse-response, and the well will need increasingly more time to reach steady state as the limit to the unstable regime or severe slugging is approached.

Validity of Stability Heuristic. In the previous sections, the stability of an equilibrium was determined by the heuristic of the direction the steady-state curves of the operating envelope intersects. This stability heuristic, however, is only able to detect static instabilities. A more rigorous way of determining stability is to use the well-known Nyquist stability criterion (see, for example, Åström and Murray 2010).

To use this criterion, we need to linearize the system and derive the loop-transfer function of a known stable loop. Assuming a well with constant choke opening and constant gas and liquid mass-rates entering at the bottom to be stable, we can analyze the system by breaking the loop between the reservoir and the well; see Fig. 11. In this block diagram, $G_{0}(s)$ denotes the linearized system dynamics between a gas mass-rate entering at the bottom of the well and the BHCP. By closing the loop with the IPR (Eqs. 14 and 15), we obtain the full system with the boundary conditions discussed previously. Hence, we can evaluate the stability of this system by using the Nyquist criterion on the loop-

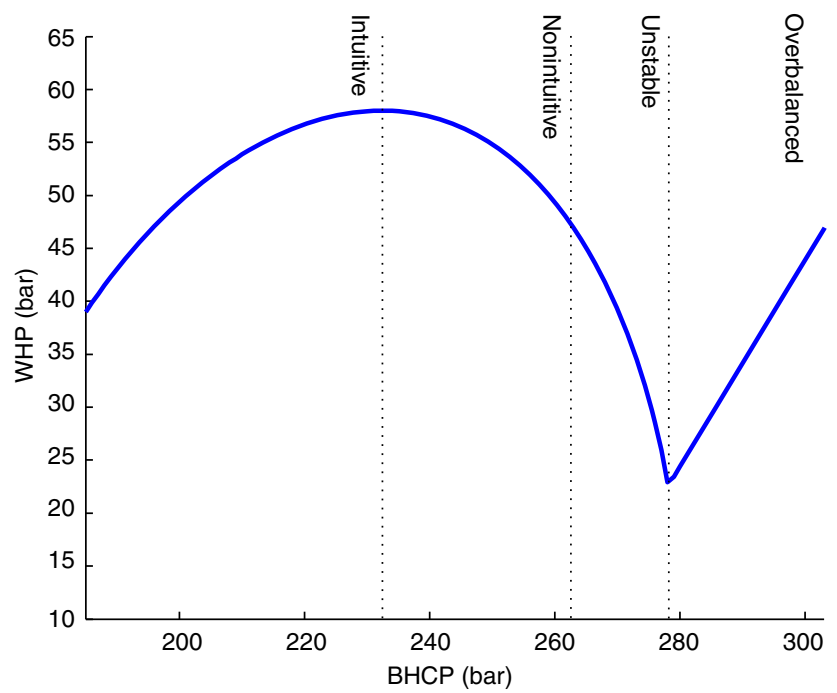

Fig. 10-The four distinct operating regimes. 


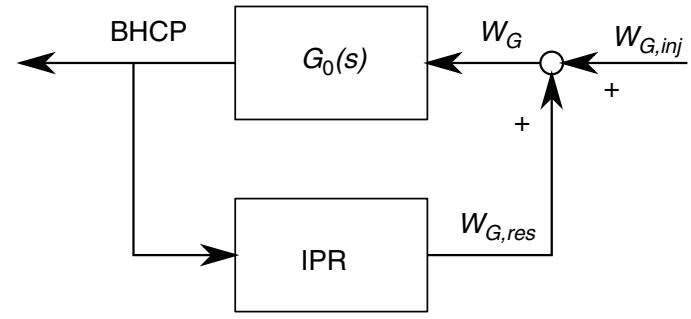

Fig. 11-Block diagram of the feedback loop that potentially causes instability.

transfer function of $G_{0}(s)$ multiplied with the linearized IPR, here denoted by $k_{G}$.

The loop-transfer function is derived in Appendix A, whereas the Nyquist criterion is restated in Appendix B. Using this criterion, we can investigate the validity of the heuristic. Consider again the equilibria shown in Fig. 9. The Nyquist contours corresponding to the two underbalanced equilibria are shown in Fig. 12. The equilibrium denoted as unstable has several encirclements of the -1 point (i.e., the complex number $-1+0 j$ ), indicating poles in the right-half plane and an unstable equilibrium point, thus confirming the heuristic in this case.

\section{Choke-Opening Sensitivity Analysis}

When controlling the BHCP by manipulating the opening of the backpressure valve, either through an automatic algorithm or with a "bloke on the choke" (i.e., manually), it is desirable to understand
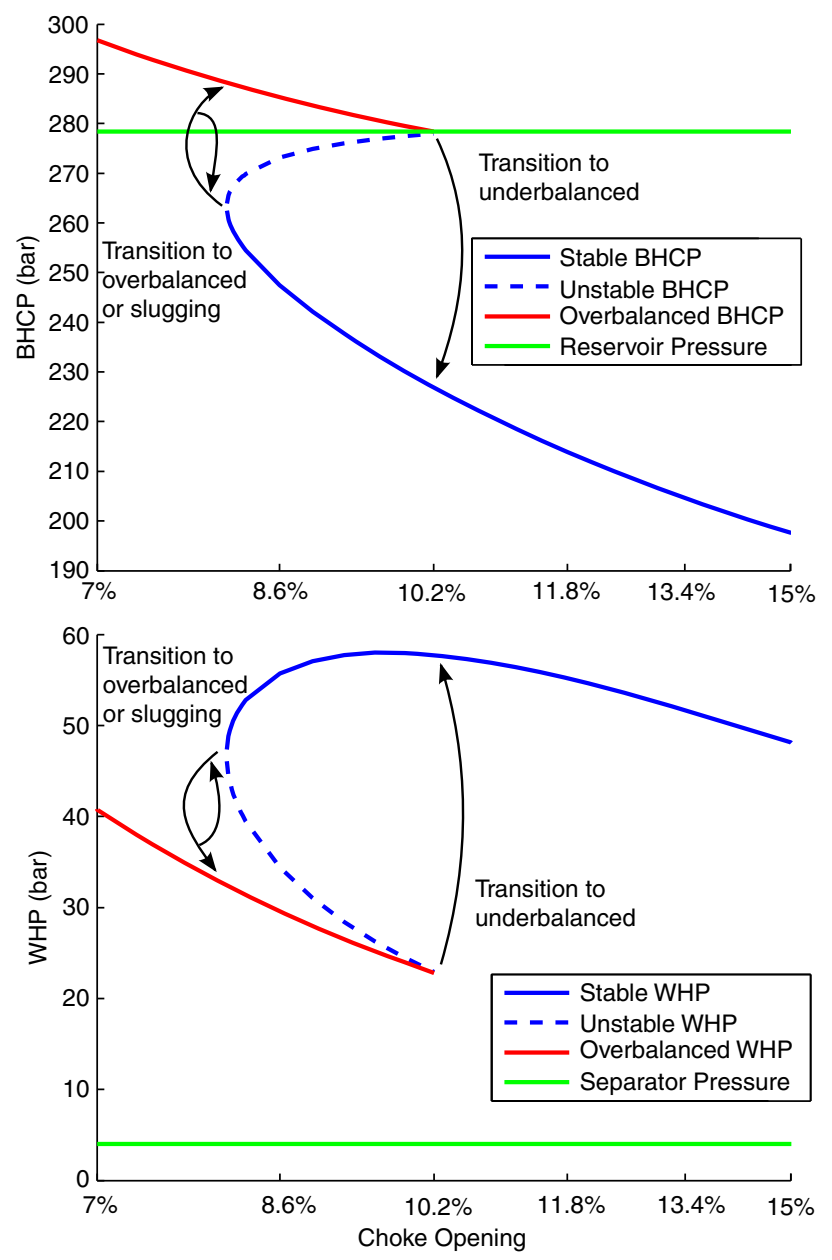

Fig. 13-Control envelope showing steady-state points plotted against choke opening. The system shows a hysteresis-like behavior in that it will converge to different steady states, depending on whether the system is over- or underbalanced.

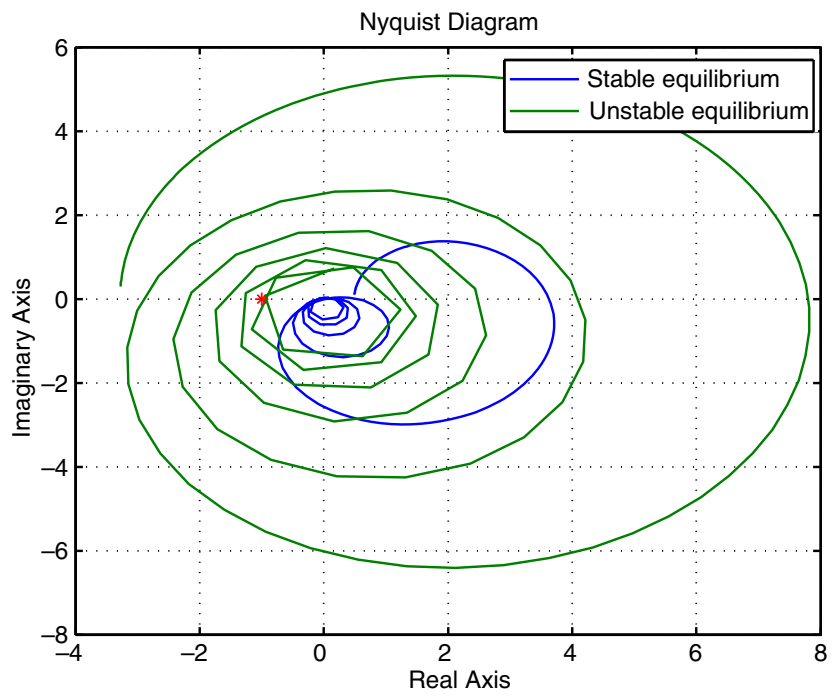

Fig. 12-Comparison of the Nyquist contours.

how the well reacts to this actuation. This is shown in Fig. 13. The steady-state values of BHCP and WHP (i.e., the green and red dots in Fig. 9) are plotted over a range of choke openings given on the $x$-axis.

Consider a well initially in the overbalanced regime. Closing the choke will cause the system to move along the red line until a choke opening of $8 \%$. This corresponds to a choke opening where the red dotted line in Fig. 8 is below the WHP minimum occurring at the transition to underbalanced conditions. The system will then move to the stable steady states given by the blue curve in Fig. 13. Reducing the choke opening when the system is in this state will make it move along the blue curve. The end of the blue curve, moving toward left, is the limit of the stable regime. Closing the choke past this point will either cause the system to go to the overbalanced regime or enter a severe slugging limit cycle.

\section{Dynamic Instability: Severe Slugging in UBO}

As discussed previously, an equilibrium must be checked for dynamic instabilities to guarantee a stable operating point. For the considered UBO scenario, in which the liquid-rate is constant and the gas-rate is dependent on drawdown, there have been reports from the field by Graham and Culen (2004) and Mykytiw et al. (2004) of instabilities associated with low gas-rates, leading to a limit cycle of severe slugging. (Note that by severe slugging we mean a violent, low-frequency cycling between long liquid slugs and a high gas flow rate, and not a slug flow pattern.) In recreating this behavior in simulations, it was found that the well would, in some instances, exhibit an oscillation with increasing magnitude, diverging from an equilibrium predicted to be stable by the stability heuristic, thus suggesting a dynamic instability. The system would then, over time, enter a limit cycle of severe slugging; see Fig. 14.

In the literature, we find that there can be different mechanisms for severe slugging, for example, terrain slugging (Taitel 1986; Jansen et al. 1996), casing heading (Eikrem et al. 2006, 2008), and density-wave (Hu 2004; Sinegre 2006). However, not one of these mechanisms matches exactly with the boundary conditions of constant liquid rate and drawdown-dependent gas rate, which are explored in this paper. The closest match is perhaps the dynamic instability reported by Xu and Golan (1989).

Thus, it seems that this particular mechanism for severe slugging in UBD has not been extensively studied in the literature and hence is not yet clearly understood. It is, however, known to be associated with low gas-rates, meaning it tends to occur close to the balance point. Furthermore, it seems to be caused by increases in WHP caused by gas flowing out through the choke. Thus, keeping WHP constant would remove the slugging but would start a static instability instead if the well is in the nonintuitive regime. 


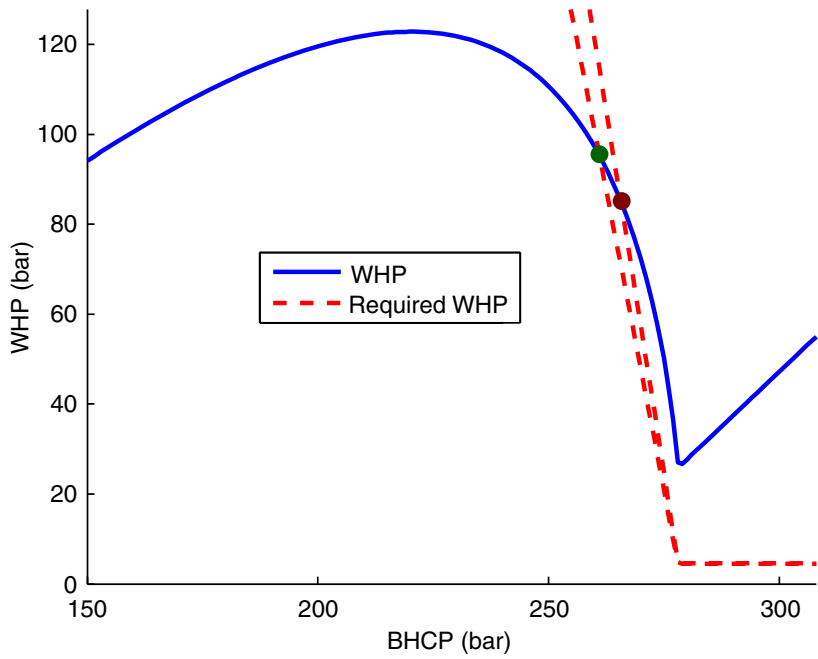

Fig. 14-Operating envelope showing the equilibria at the transition to severe slugging.

To recreate slugging with the DFM, the backpressure choke Eq. 9, with a very small $Y$, is required. The effect of this is that variations in gas flow through the choke cause large changes in WHP. The operating envelope of a well that exhibits slugging is shown in Fig. 15; here, $Y=0.025$ was used. Fig. 15 also shows the required WHP curves of two slightly different choke openings: one that corresponds to a stable equilibrium and one that has a dynamic instability. With such a low $Y$ constant, the unstable regime discussed in the previous section, caused by the static instability, is avoided because of the steepness of the required WHP curves. Instead, the occurrence of severe slugging becomes possible. To evaluate the stability of these equilibria, we again find the loop-transfer function with the method described in Appendix A and the Nyquist theorem from Appendix B.

The loop-transfer functions and the Nyquist contours of the linearized systems obtained around the two equilibria are shown in Fig. 16. The loop-transfer function shows the frequencydomain response of the system to an impulse of increased gas influx. In the Nyquist plot, we see that the slugging instability in this case is caused by two unstable modes in the $2 \times 10^{-3}-1 \times 10^{-2}$ $(\mathrm{rad} / \mathrm{s})$ frequency range. This is consistent with the transient simulation shown in Fig. 16 in which the slugging has approximately a 20 -minute period, which corresponds to $5 \times 10^{-3}(\mathrm{rad} / \mathrm{s})$.
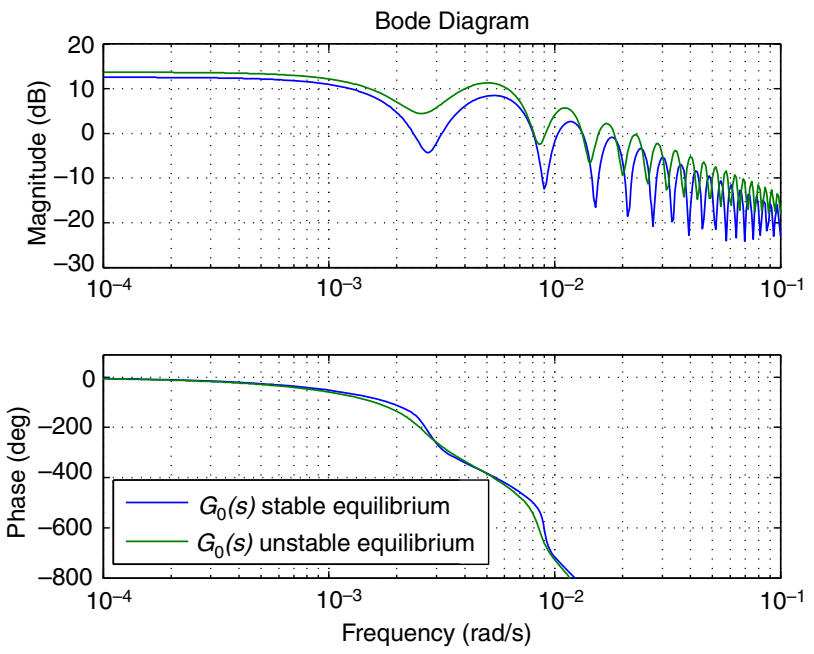

\section{Summary and Conclusions}

In this paper, an operating-envelope technique for analyzing dynamics encountered in UBO is presented. Using this technique, we are able to identify five distinct operating regimes:

- Intuitive regime-with short and intuitive transient dynamics, associated with high gas-rates.

- Nonintuitive regime - with an inverted WHP response, associated with low-to-midrange gas rates.

- Unstable regime, static instability - with no stable steady states because of a static instability, associated with low gas rates.

- Unstable regime, severe slugging — with no stable steady states because of the well tending to a severe slugging limit cycle, caused by dynamic instability, associated with low gas rates.

- Overbalanced regime-with no reservoir influx and stable steady states.

The accompanying analysis reveals that an important factor deciding the behavior of an underbalanced gas well is not whether the well is frictionally or hydrostatically dominated, but instead the limit between the intuitive and nonintuitive regimes, given by the point when

$$
\frac{1}{k_{G}}+\frac{\Delta F}{\Delta W_{G}}+\frac{\Delta G}{\Delta W_{G}}=0 .
$$

Another important determining factor for the behavior is the limit to the two unstable regimes. It can be shown that both a steep and flat slope of the "Required WHP" curve of the backpressure choke can cause instabilities:

- A static instability in the case of a flat curve (see Figs. 9 and 12).

- A dynamic instability causing severe slugging in the case of a steep curve (see Figs. 15 and 16).

The location of the limits to these two regimes will typically be uncertain, but it was shown that they are both associated with low gas rates, and some insight can be gained by deriving the loop-transfer function and using the Nyquist stability criterion, as described in Appendices B and C.

Potential for Automatic Control. The analysis and classification provided in this paper could serve as a decision support tool to identify operating conditions and anticipate the behavior of the system. This should allow operators to avoid counterproductive operation of the choke, as is reported in Graham and Culen (2004).

An even more promising line of future research, however, is the potential for automatic control. There are many examples from other industries in which feedback control was used to turn a nominally unstable equilibrium into a stable one (see, for example, Krstic et al. 1995), hence making it a possible operating point.

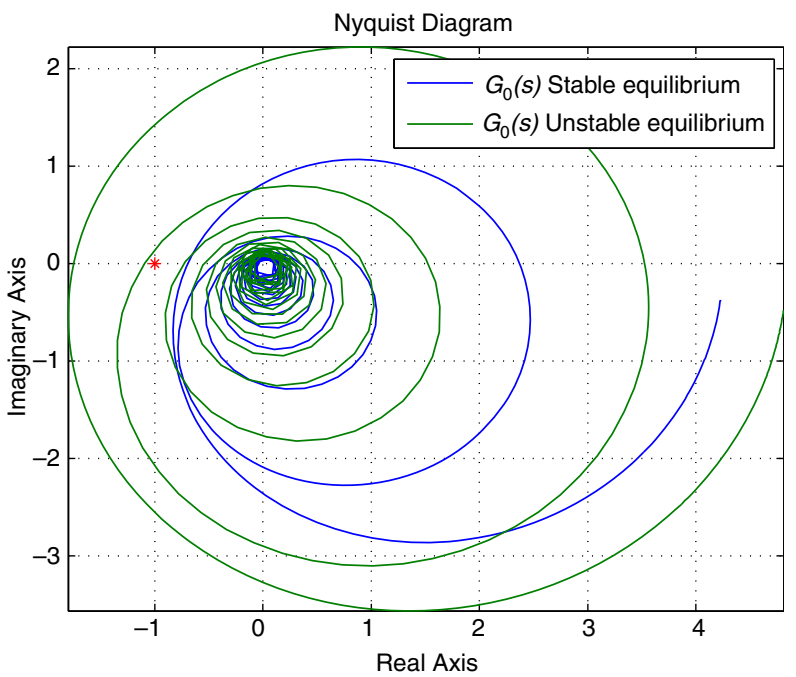

Fig. 15-Loop transfer functions (left) and Nyquist plot (right) of well entering a severe slugging limit cycle by decreasing the choke opening. 

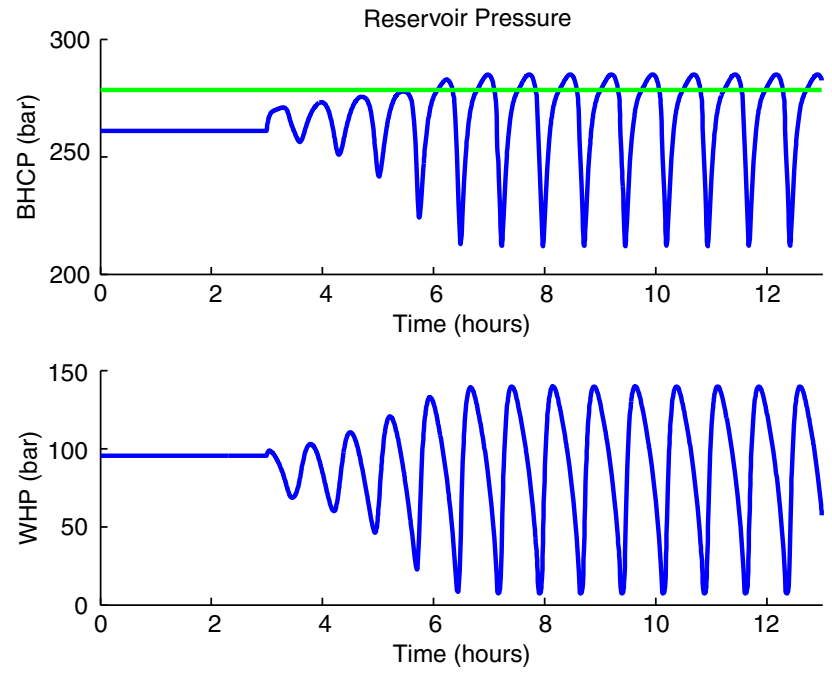

Fig. 16-Transient simulation of the well entering a slugging limit cycle when changing choke opening at 3 hours.

By stabilizing the unstable regions with feedback control, UBO could be performed arbitrarily close to the balance point, thus avoiding excessive flaring of produced gas and enabling UBD of wells with low collapse margins. Conceptually, stabilizing an underbalanced well can be understood as analogous to controlling a pendulum at the upper equilibrium: straightforward to do with an actuator controlled with feedback from position measurements, but possibly challenging and tiring if performed manually. This topic will be investigated in future publications.

\section{Acknowledgments}

This work was supported by Statoil ASA and the Norwegian Research Council (NFR project 210432/E30 Intelligent Drilling).

\section{References}

Aarsnes, U. J. F., Aamo, O. M., Hauge, E. et al. 2013. Limits of Controller Performance in the Heave Disturbance Attenuation Problem. In Control Conf. (ECC), 2013 European, 1071-1076. Zürich: IEEE.

Aarsnes, U. J. F., Di Meglio, F., Aamo, O. M. et al. 2014a. Fit-for-Purpose Modeling for Automation of Underbalanced Drilling Operations. Presented at the SPE/IADC Managed Pressure Drilling and Underbalanced Operations Conference and Exhibition, Madrid, Spain, 8-9 April. SPE-168955-MS. http://dx.doi.org/10.2118/168955-MS.

Aarsnes, U. J. F., Di Meglio, F., Evje, S. et al. 2014b. Control-Oriented Drift-Flux Modeling of Single- and Two-Phase Flow for Drilling. In ASME Dyn. Syst. Control Conf., page V003T37A003, San Antonio, Texas, USA: ASME.

Aarsnes, U. J. F., Gleditsch, M. S., Aamo, O. M. et al. 2014c. Modeling and Avoidance of Heave-Induced Resonances in Offshore Drilling. SPE Drill Compl 29 (4): 454-464. SPE-173178-PA. http://dx.doi.org/ 10.2118/173178-PA.

Åström, K. J. and Murray, R. M. 2010. Feedback Systems: An Introduction for Scientists and Engineers. Princeton University Press.

Di Meglio, F. 2011. Dynamics and Control of Slugging in Oil Production. $\mathrm{PhD}$ thesis, ParisTech.

Eikrem, G. O., Aamo, O. M., and Foss, B. 2006. Stabilization of Gas-Distribution Instability in Single-Point Dual Gas Lift Wells. SPE Prod \& Oper 21 (2): 252-259. SPE-97731-PA. http://dx.doi.org/10.2118/ 97731-PA.

Eikrem, G. O., Aamo, O. M., and Foss, B. 2008. On Instability in Gas Lift Wells and Schemes for Stabilization by Automatic Control. SPE Prod \& Oper 23 (2): 268-279. SPE-101502-PA. http://dx.doi.org/10.2118/ 101502-PA.

Evje, S. and Fjelde, K. K. 2002. Hybrid Flux-Splitting Schemes for a Two-Phase Flow Model. J. Comput. Phys. 175 (2): 674-701. http:// dx.doi.org/10.1006/jcph.2001.6962.
Evje, S. 2011. Weak Solutions for a Gas-Liquid Model Relevant for Describing Gas-Kick in Oil Wells. SIAM J. Math. Anal. 43 (4): 1887-1922. http://dx.doi.org/10.1137/100813932.

Fjelde, K. K., Rommetveit, R., Merlo, A. et al. 2003. Improvements in Dynamic Modeling of Underbalanced Drilling. Presented at the IADC/ SPE Underbalanced Technology Conference and Exhibition, Houston, 25-26 March. SPE-81636-MS. http://dx.doi.org/10.2118/81636-MS.

Godhavn, J.-M. 2010. Control Requirements for Automatic Managed Pressure Drilling System. SPE Drill Compl 25 (3): 336-345. SPE-119442PA. http://dx.doi.org/10.2118/119442-PA.

Godhavn, J.-M. 2011. Drilling Seeking Automatic Control Solutions. In 18th IFAC World Congr. Milano, ed. B. Sergio, 10842-10850, Milano, Italy.

Graham, R. A. and Culen, M. S. 2004. Methodology for Manipulation of Wellhead Pressure Control for the Purpose of Recovering Gas To Process in Underbalanced Drilling Applications. Presented at the SPE/ IADC Underbalanced Technology Conference and Exhibition, Houston, 11-12 October. SPE-91220-MS. http://dx.doi.org/10.2118/91220MS.

Guo, B. 2002. Balance Between Formation Damage and Wellbore Damage: What Is the Controlling Factor in UBD Operations? Presented at the International Symposium and Exhibition on Formation Damage Control, Lafayette, Louisiana, 20-21 February. SPE-73735-MS. http:// dx.doi.org/10.2118/73735-MS.

Guo, B. and Ghalambor, A. 2002. An Innovation in Designing Underbalanced Drilling Flow Rates: A Gas-Liquid Rate Window (GLRW) Approach. Presented at the IADC/SPE Asia Pacific Drilling Technology, Jakarta, 8-11 September. SPE-77237-MS. http://dx.doi.org/10.2118/77237-MS.

Hu, B. 2004. Characterizing Gas-Lift Instabilities. Trondheim, Norway: NTNU.

Ishii, M. 1977. One-dimensional drift-flux model and constitutive equations for relative motion between phases in various two-phase flow regimes. Technical report, Argonne, Illinois: Argonne National Laboratory (ANL).

Jansen, F., Shoham, O., and Taitel, Y. 1996. The Elimination of Severe Slugging Experiments and Modeling. Int. J. Multiph. Flow 22 (6): 1055-1072. http://dx.doi.org/10.1016/0301-9322(96)00027-4.

Khalil, H. K. 2002. Nonlinear Systems, third edition. Upper Saddle River: Prentice Hall.

Krstic, M., Protz, J., Paduano, J. et al. 1995. Backstepping Designs for Jet Engine Stall and Surge Control. In Proc., 1995 34th IEEE Conf. Decision Control, Vol. 3, pages 3049-3055. http://dx.doi.org/10.1109/ CDC.1995.478612.

Lage, A., Fjelde, K., and Time, R. 2000. Underbalanced Drilling Dynamics: Two-Phase Flow Modeling and Experiments. In Proc., IADC/SPE Asia Pacific Drill. Technol., Vol. 8, pages 61-70, Kuala Lumpur, Malaysia. Society of Petroleum Engineers.

Lage, A. and Time, R. 2002. An Experimental and Theoretical Investigation of Upward Two-Phase Flow in Annuli. SPE J. 7 (3): 16-18. SPE79512-PA. http://dx.doi.org/10.2118/79512-PA.

Mykytiw, C., Davidson, I., and Frink, P. 2003. Design and Operational Considerations to Maintain Underbalanced Conditions With Concentric Casing Injection. Presented at the IADC/SPE Underbalanced Technology Conference and Exhibition, Houston, USA, 25-26 March. SPE-81631-MS. http://dx.doi.org/10.2118/81631-MS.

Mykytiw, C., Suryanarayana, P., and Brand, P. 2004. Practical Use of a Multiphase Flow Simulator for Underbalanced Drilling Applications Design-The Tricks of the Trade. Presented at the SPE/IADC Underbalanced Technology Conference and Exhibition, Houston, USA, 11-12 October. SPE-91598-MS. http://dx.doi.org/10.2118/91598-MS.

Nguyen, C., Somerville, J., and Smart, B. 2009. Predicting the Production Capacity During Underbalanced-Drilling Operations in Vietnam. Presented at the IADC/SPE Managed Pressure. Drilling and Underbalanced Operations Conference and Exhibition, San Antonio, Texas, USA, 12-13 February. SPE-122266-MS. http://dx.doi.org/10.2118/122266-MS.

Pickles, R., Brand, P., and Savage, P. 2004. Utilization of Underbalanced Drilling Techniques to Exploit a Low-Pressure Reservoir in Indonesia. Presented at the SPE/IADC Underbalanced Technology Conference and Exhibition, Houston, USA, 11-12 October. SPE-91591-MS. http://dx.doi.org/10.2118/91591-MS.

Saponja, J. 1998. Challenges With Jointed-Pipe Underbalanced Operations. SPE Drill \& Compl 13 (2): 121-128. SPE-37066-PA. http:// dx.doi.org/10.2118/37066-PA. 
Shi, H., Holmes, J., Durlofsky, L. et al. 2005. Drift-Flux Modeling of Two-Phase Flow in Wellbores. SPE J. 10 (1): 24-33. SPE-84228-PA. http://dx.doi.org/10.2118/84228-PA.

Sinegre, L. 2006. Dynamic Study of Unstable Phenomena Stepping in Gas-Lift Activated Systems, PhD thesis, MINES ParisTech.

Suryanarayana, P., Smith, B. E., Hasan, A. K. et al. 2006. Basis of Design for Coiled-Tubing Underbalanced Through-Tubing Drilling in the Sajaa Field. SPE Drill Compl 21 (2): 125-132. SPE-87146-PA. http:// dx.doi.org/10.2118/87146-PA.

Taitel, Y. 1986. Stability of Severe Slugging. Int. J. Multiph. Flow 12 (2): 203-217. http://dx.doi.org/10.1016/0301-9322(86)90026-1.

Thorogood, J., Aldred, W. D., Florence, F. et al. 2010. Drilling Automation: Technologies, Terminology, and Parallels With Other Industries. SPE Drill Compl 25 (4): 419-425. SPE-119884-PA. http://dx.doi.org/ 10.2118/119884-PA.

Udegbunam, J. E., Fjelde, K. K., Arild, O. Y. et al. 2013. UncertaintyBased Approach for Predicting the Operating Window in UBO Well Design. SPE Drill Compl 28 (4): 326-337. SPE-164916-PA. http:// dx.doi.org/10.2118/164916-PA.

Udegbunam, J. E., Fjelde, K. K. R., Evje, S. et al. 2014. A Simple Transient Flow Model for MPD and UBD Applications. Presented at the SPE/IADC Managed Pressure Drilling \& Underbalanced Operations Conference \& Exhibition, Madrid, Spain, 8-9 April. SPE-168960-MS. http://dx.doi.org/10.2118/168960-MS.

Wallis, G. B. 1969. One-Dimensional Two-Phase Flow. New York: McGraw-Hill.

Wiggins, M., Russell, J., and Jennings, J. 1996. Analytical Development of Vogel-Type Inflow Performance Relationships. SPE J. 1 (4): 355-362. SPE-23580-PA. http://dx.doi.org/10.2118/23580-PA.

Xu, Z. G. and Golan, M. 1989. Criteria for Operation Stability of Gas-Lift Wells. (unsolicited). SPE-19362-PA. http://dx.doi.org/10.2118/19362-PA.

\section{Appendix A: The DFM}

The following is a recap of the formulation described by Aarsnes et al. (2014b) and Udegbunam et al. (2014) on the basis of the work by Evje and Fjelde (2002).

Distributed Equations. In this version of the DFM, the mud, oil, and water are lumped into a single liquid phase. In developing the model, we use the following mass variables:

$$
m=\alpha_{L} \rho_{L}, \quad n=\alpha_{G} \rho_{G},
$$

where for $k=L, G$ denoting liquid or gas, $\rho_{k}$ is the phase density, and $\alpha_{k}$ the volume fraction satisfying

$$
\alpha_{L}+\alpha_{G}=1
$$

Further $v_{k}$ denotes the velocities, and $P$ the pressure. All these variables are functions of time and space. We denote $t \geq 0$ the time variable, and $x \in[0, L]$ the space variable, corresponding to a curvilinear abscissa with $x=0$ corresponding to the bottom hole and $s=L$ to the outlet choke position (see Fig. 2). The equations are as follows:

$$
\begin{aligned}
& \frac{\partial m}{\partial t}+\frac{\partial m v_{L}}{\partial x}=0, \ldots \ldots \ldots \ldots \ldots \ldots \ldots \ldots \ldots \ldots \ldots \ldots \ldots \ldots \ldots \\
& \frac{\partial n}{\partial t}+\frac{\partial n v_{G}}{\partial x}=0, \quad \ldots \ldots \ldots \ldots v_{L}^{2} \\
& \frac{\partial m v_{L}+n v_{G}}{\partial t}+\frac{\partial P+m \ldots}{\partial x} \\
& =-(m+n) g \sin \phi(x)-\frac{2 f(m+n) v_{m}\left|v_{m}\right|}{D} .
\end{aligned}
$$

In the momentum equation (Eq. A-5), the term $(m+$ $n) g \sin \phi(x)$ represents the gravitational source term, whereas $-\frac{2 f(m+n) v_{m}\left|v_{m}\right|}{D}$ accounts for frictional losses. The mixtures velocity is given as

$$
v_{m}=\alpha_{G} v_{G}+\alpha_{L} v_{L} .
$$

Along with these distributed equations, algebraic relations are needed to close the system.

Closure Relations. Both the liquid and gas phases are assumed compressible. This is required for the model to handle the transition from two-phase to single-phase flow. The densities are thus given as functions of the pressure as follows:

$$
\rho_{G}=\frac{P}{c_{G}^{2}(T)}, \quad \quad \rho_{L}=\rho_{L, 0}+\frac{P}{c_{L}^{2}}, \ldots \ldots
$$

where $c_{k}$ is the velocity of sound and $\rho_{L, 0}$ is the reference density of the liquid phase given at vacuum. Notice that the velocity of sound in the gas phase $c_{G}$ depends on the temperature, as suggested by the ideal gas law. The temperature profile is assumed to be known and is fed into the model.

Because the momentum equation (Eq. A-5) was written for the gas/liquid mixture, a so-called slip law is needed to empirically relate the velocities of gas and liquid. To handle the transition between single- and two-phase flows, we need a relation with state-dependent parameters (Shi et al. 2005; Evje 2011). More precisely, we use the following slip law:

$$
v_{G}=\left[K-(K-1) \alpha_{G}\right] v_{m}+\alpha_{L} S,
$$

where $K \geq 1$ and $S \geq 0$ are constant parameters.

\section{Appendix B: Loop-Transfer Function Derivation}

The goal here is to develop the systems-transfer functions of various input/output configurations of interest. To achieve this, following the lines of Aarsnes et al. (2013, 2014c), we linearize the system around a steady-state profile and take the Laplace transform of the system with regard to time. We then obtain a thirdorder linear, but space-variant, ordinary-differential equation (ODE) in space. This ODE must be solved for its boundary conditions. There are two boundary conditions on the left (downhole) boundary and one on the right (topside). Hence, we must obtain the transition matrix to transport the basis of the solution from the right side to the left (or vice versa) to obtain the specific solution.

Linearization. The system of Eqs. A-3 through A-5 with boundary conditions given by Eqs. 6 through 9 can be written in the quasilinear form

$$
\frac{\partial q(x, t)}{\partial t}+A[q(x, t)] \frac{\partial q(x, t)}{\partial x}=S[q(x, t)], \ldots \ldots \ldots
$$

with boundary conditions

$$
h_{1}[q(0, t), U(t)]=h_{2}[q(0, t), U(t)]=h_{3}[q(L, t), V(t)]=0,
$$

where $q=\left[\begin{array}{lll}m & n & I\end{array}\right]$ is the state vector and the actuation acts through the exogenous variables $U(t)=\left[\begin{array}{ll}W_{L, \text { inj }} & W_{G, \text { inj }}\end{array}\right]^{T}$ and $V(t)=Z$.

Let $\tilde{q}(x, t)=q(x, t)-\bar{q}(x)$ denote the distance from some equilibrium profile $\bar{q}$. Close to this equilibrium profile, the dynamics of the system can be approximated by the linear system (Di Meglio 2011):

$$
\frac{\partial \tilde{q}(x, t)}{\partial t}+A(\bar{q}(x)) \frac{\partial \tilde{q}(x, t)}{\partial x}=\left[-\frac{\partial A}{\partial q} \cdot \bar{q}^{\prime}(x)+\frac{\partial S}{\partial q}\right]_{q=\bar{q}} \tilde{q}(x, t),
$$$$
\ldots \ldots \ldots \ldots \ldots(\mathrm{B}-3)
$$

where we have used the fact that the equilibrium satisfies

$$
A(\bar{q}) \bar{q} \prime(x)=S(\bar{q})
$$


Also, note the notation:

$$
\left.\frac{\partial A}{\partial q} \cdot \bar{q}^{\prime}(x)\right|_{q=\bar{q}}=\left[\frac{\partial A}{\partial m} \bar{m}^{\prime}(x)+\frac{\partial A}{\partial n} \bar{n}^{\prime}(x)+\frac{\partial A}{\partial I} \bar{I}^{\prime}(x)\right]_{q=\bar{q}} .
$$

Taking the Laplace transform of Eq. B-3 in time, denoting the Laplace variable by $s$, we get the following ODE in space:

$$
\begin{aligned}
& \frac{\partial \tilde{q}(x, s)}{\partial x}=\bar{A}(x, s) \tilde{q}(x, s), \ldots \ldots \ldots \ldots \ldots \\
& \bar{A}(x, s) \equiv A^{-1}(\bar{q})\left[-\frac{\partial A}{\partial q} \cdot \bar{q} \prime(x)+\frac{\partial S}{\partial q}-s I^{3 \times 3}\right]_{q=\bar{q}} \ldots
\end{aligned}
$$

Numerical Solutions. Because Eq. B-6 is a linear ODE, we can superimpose solutions to construct the transition matrix. This is performed by solving Eq. B-6 from $x_{0}$ to $x_{1}$ three times with the initial conditions:

$$
\tilde{q}^{1}\left(x_{0}, s\right)=\left[\begin{array}{l}
1 \\
0 \\
0
\end{array}\right], \quad \tilde{q}^{2}\left(x_{0}, s\right)=\left[\begin{array}{l}
0 \\
1 \\
0
\end{array}\right], \quad \tilde{q}^{3}\left(x_{0}, s\right)=\left[\begin{array}{l}
0 \\
0 \\
1
\end{array}\right]
$$

The solutions, denoted as $\tilde{q}^{1}\left(x_{1}, s\right), \tilde{q}^{2}\left(x_{1}, s\right), \tilde{q}^{3}\left(x_{1}, s\right)$, make up the transition matrix:

$$
\Phi\left(x_{1}, x_{0}\right)=\left[\tilde{q}^{1}\left(x_{1}\right) \tilde{q}^{2}\left(x_{1}\right) \tilde{q}^{3}\left(x_{1}\right)\right]
$$

which has the property

$$
\tilde{q}\left(x_{1}, s\right)=\Phi\left(x_{1}, x_{0}\right) \tilde{q}\left(x_{0}, s\right)
$$

Boundary Conditions. Consider the following linearization of the boundary conditions:

$$
\begin{aligned}
& \frac{\partial h_{1}}{\partial q} \tilde{q}(0, s)=-\frac{\partial h_{1}}{\partial U} \tilde{U}(s), \\
& \frac{\partial h_{2}}{\partial q} \tilde{q}(0, s)=-\frac{\partial h_{2}}{\partial U} \tilde{U}(s), \\
& \frac{\partial h_{3}}{\partial q} \tilde{q}(1, s)=-\frac{\partial h_{3}}{\partial V} \tilde{V}(s),
\end{aligned}
$$

where the partial derivatives are evaluated at $q=\bar{q}, U=\bar{U}$, $V=\bar{V}$. Using the derived transition matrix, we can write these in matrix form

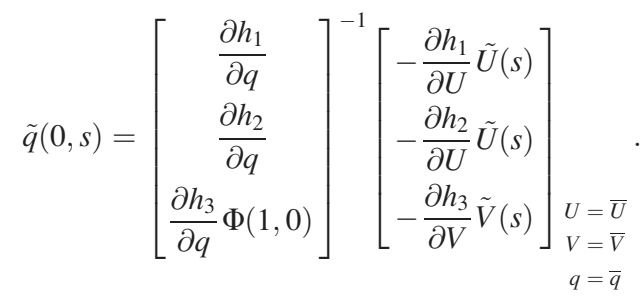

The states at other positions can be obtained with Eq. B-10.
Transfer Functions. Finally, we can obtain the desired transfer functions. Considering the output $g(q)$ with perturbations denoted $\tilde{g}[q(0, s)]=g[q(0, s)]-g[\bar{q}(0)]$, the transfer function from, for example, the choke input $Z(s)=V(s)$ is given as

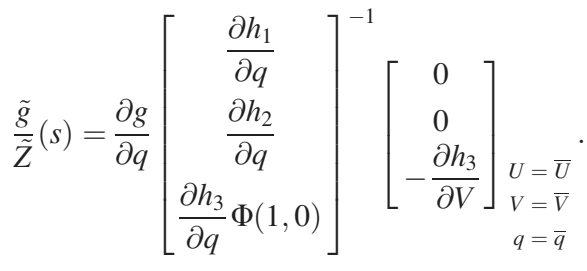

\section{Appendix C: The Nyquist Stability Criterion}

Assuming that for constant influx rates of gas and liquid the DFM is stable, the cause for the potential instability must be the interaction between the dynamics in the well and the IPR; see Fig. 11.

Stability of an equilibrium point can be determined by checking the stability of the linearized system, as per Lyapunov's indirect method (Khalil 2002). Hence, we can determine the stability of equilibria encountered in UBD by using the Nyquist criterion on the loop-transfer function developed in Appendix A. A restatement of the Nyquist criterion is as follows (Åström and Murray 2010):

Theorem 1. Let $G_{0}(s) k_{G}$ be the loop transfer function for a negative feedback system (as shown in Fig. 11) and assume that $G_{0}(s) k_{G}$ has no poles in the closed right half-plane $(\operatorname{Re} s \geq 0)$ except for single poles on the imaginary axis. Then the closed loop system is stable if and only if the closed contour given by $\Omega=\left\{G_{0}(j \omega) k_{G}: \infty<\omega<\infty\right\} \subset \mathbb{C}$ has no net encirclements of the critical point $s=-1$.

Ulf Jakob Flø Aarsnes is a PhD degree student at the Norwegian University of Science and Technology (NTNU) from which he also holds an MSc in engineering cybernetics. His research interests include modeling, analysis, and control of distributed parameter systems and their application to drilling. Aarsnes has authored or coauthored more than 15 technical papers in this field. He is a member of SPE.

Florent Di Meglio is an associate professor at the Centre Automatique et Systèmes at MINES ParisTech. He has been there for 3 years. Di Meglio's current interests include multiphase flow, drilling, process control, estimation theory, and partialdifferential equations. He holds a PhD degree in mathematics and contol from MINES ParisTech.

Robert Graham holds a degree in mechanical engineering. $\mathrm{He}$ is Technical Director of Well Advanced Solutions and a Chief Adviser and Partner with Kelda Drilling Controls. Graham is also a Senior Drilling Adviser for MPD/UBD at Shell UK and acting MPD Subject Matter Expert for Shell Global Technology. He has held various leading technical and managerial positions in several oil companies and service companies in the drilling industry, including Mobil Oil, Fracmaster, Schlumberger, Precision Drilling, DONG Energy, Statoil, and Shell. Graham has specialized in advanced drilling techniques such as coiled-tubing drilling (CTD), UBD, and MPD during the past $20+$ years and has significant technical, management, and operational experience from state-of-the-art MPD and UBD projects worldwide. He has authored a dozen papers on MPD, UBD, and CTD and holds a patent in the MPD field.

Ole Morten Aamo earned MSc and PhD degrees in engineering cybernetics from NTNU, Trondheim, Norway. He is currently a professor with NTNU. His research interests include control of distributed parameter systems with special emphasis on control of fluid flows. Aamo is a coauthor of the book Flow Control by Feedback (Springer-Verlag 2003). 